\title{
Implizite Fähigkeitstheorien, akademisches Selbstkonzept und schulisches Wohlbefinden
}

\author{
Marko Lüftenegger (D) · Julia Holzer (D) Barbara Schober
}

Eingegangen: 14. April 2021 / Überarbeitet: 12. September 2021 / Angenommen: 20. September 2021 /

Online publiziert: 13. Oktober 2021

(C) Der/die Autor(en) 2021

Zusammenfassung Implizite Fähigkeitstheorien haben hohe Relevanz für Lernverhalten und Leistung von Schüler*innen. Diese Effekte werden in kognitiven Bedeutungssystemen durch selbstbezogene Kompetenzeinschätzungen vermittelt. Neben Lernverhalten und Leistung rückte als weiteres zentrales Ziel von Schule und Unterricht in den letzten Jahren auch zunehmend schulisches Wohlbefinden in den Fokus. Studien zu Zusammenhängen von Impliziten Theorien und Wohlbefinden sind jedoch rar. Vorhandene Arbeiten betrachteten Wohlbefinden dabei überwiegend als die Abwesenheit von pathologischen Merkmalen, während neuere Ansätze das Vorhandensein von Ressourcen und die Multidimensionalität von Wohlbefinden im Jugendalter betonen. Ein solches Modell ist das EPOCH-Modell welches die Dimensionen Engagement, Perseverance, Optimism, Connectedness und Happiness unterscheidet.

Die vorliegende Studie untersucht Zusammenhänge zwischen inkrementellen Impliziten Fähigkeitstheorien, akademischem Selbstkonzept und Wohlbefinden von Schüler*innen. Wohlbefinden wird dabei als kontextspezifisches Konstrukt - adaptiert an den Schulkontext - durch die fünf EPOCH Dimensionen operationalisiert.

Die Stichprobe der Fragebogenstudie umfasste 1484 österreichische Schüler*innen (52\% weiblich; $\mathrm{M}=12,95 ; \mathrm{SD}=2,10)$ aus 87 Klassen. Zur Untersuchung der Zusammenhänge wurde ein latentes Mediationsmodell mit inkrementeller Fähigkeitstheorie als unabhängiger Variable, Selbstkonzept als Mediator und Wohlbefinden als abhängiger Variable spezifiziert. Die Ergebnisse zeigten positive Zusammenhänge von inkrementeller Fähigkeitstheorie mit Selbstkonzept sowie mit

Marko Lüftenegger $(\varangle) \cdot$ Julia Holzer $\cdot$ Barbara Schober

Institut für Psychologie der Entwicklung und Bildung, Fakultät für Psychologie, Universität Wien,

Universitätsstraße 7 (NIG), 1010 Wien, Österreich

E-Mail: marko.lueftenegger@univie.ac.at

Marko Lüftenegger

Institut für LehrerInnenbildung, Zentrum für LehrerInnenbildung, Universität Wien,

Porzellangasse 4, 1090 Wien, Österreich 
allen fünf EPOCH Dimensionen. Die Effekte von inkrementeller Fähigkeitstheorie auf Engagement, Perseverance und Optimism werden durch das Selbstkonzept mediiert. Limitationen dieser Studie sowie Implikationen für Theorie, Praxis und zukünftige Forschung werden diskutiert.

Schlüsselwörter Wohlbefinden · Implizite Theorien · Selbstkonzept · Schule · Mindset · EPOCH

\section{Implicit theories of ability, academic self-concept and well-being in school}

Abstract Implicit theories of ability are important factors for students' adaptive learning behavior and performance. These effects are mediated in cognitive meaning systems through competence self-perceptions. In addition to learning behavior and performance, well-being has increasingly come into focus recently as a central goal of schooling and instruction. However, studies on the association between implicit theories and well-being are scarce. Existing work has predominantly considered well-being as the absence of pathological features, whereas more recent approaches emphasize the presence of resources and the multidimensionality of well-being in adolescence. One such model is the EPOCH model with the dimensions engagement, perseverance, optimism, connectedness, and happiness.

This study examines relationships between incremental implicit theory of ability, academic self-concept, and student well-being. Well-being, adapted to the school context, is operationalized as a context-specific construct by the five EPOCH dimensions.

The sample of the questionnaire study comprised 1484 Austrian students (52\% female; $\mathrm{M}=12.95 ; \mathrm{SD}=2.10$ ) from 87 classes. To examine the relationships, a latent mediation model was specified with incremental ability theory as the independent variable, self-concept as the mediator, and well-being as the dependent variable. The results showed positive relations of incremental ability theory with self-concept as well as with all five EPOCH dimensions. The effects of incremental ability theory on engagement, perseverance, and optimism were mediated by self-concept. Limitations of this study as well as implications for theory, practice, and future research are discussed.

Keywords Well-being · Implicit theories · Self-concept · Secondary school · EPOCH $\cdot$ Mindset

\section{Einleitung}

Um ihre Welt zu erklären und $\mathrm{zu}$ verstehen entwickeln Individuen grundlegende Annahmen über menschliche Eigenschaften. Diese Impliziten Theorien (Dweck und Leggett 1988), in neuerer Forschung auch als Mindsets bezeichnet (Dweck 2006; Dweck und Yeager 2019), beinhalten fundamentale Überzeugungen über die Veränderbarkeit (Inkrementalität) und Stabilität (Entität) von menschlichen Eigenschaf- 
ten wie Intelligenz, Fähigkeiten, Willenskraft und Persönlichkeit (Lüftenegger und Chen 2017b). Diese Überzeugungen entwickeln sich unter anderem durch schulische Sozialisation (Dweck 2007) und können in sensiblen Phasen wie der pubertären Reifung eine wichtige Rolle spielen (Dahl et al. 2018; Yeager et al. 2018). Für die Adoleszenz zeigen Forschungsbefunde, dass diese Theorien über das eigene Selbst mit akademischer Leistung (Burnette et al. 2013; West et al. 2018; OECD 2019; Sisk et al. 2018), selbstbezogenen Zielen (Lüftenegger et al. 2015), Emotionen (Burnette et al. 2013) sowie psychischer Gesundheit (Schleider et al. 2015; Burnette et al. 2020) in Zusammenhang stehen. In der Gesundheitsforschung wurden Entitätstheorien bei Jugendlichen allerdings hauptsächlich mit Stress und Depression in Verbindung gebracht (Burnette et al. 2020; Schleider et al. 2015). Eine robuste Befundlage für den Zusammenhang mit positiven Aspekten wie Wohlbefinden fehlt bisher (für eine Ausnahme siehe King 2017).

Dies könnte darin begründet liegen, dass die Forschung zum psychologischen Wohlbefinden im Vergleich zu anderen Konstrukten ein relativ junges Forschungsfeld in der Psychologie und insbesondere in der Pädagogischen Psychologie darstellt und sich zunächst hauptsächlich auf Erwachsene konzentrierte (Deci und Ryan 2008; Diener 1984; Ryan und Deci 2001). Empirische Studien mit Jugendlichen zeigen allerdings bereits Zusammenhänge von Wohlbefinden mit bedeutsamen schulspezifischen Aspekten wie Selbstkontrolle bei schulbezogenen Aufgaben (Howell 2009) und Selbstwirksamkeit im Umgang mit schulbezogenen Herausforderungen (Hascher 2010). Obwohl selbstbezogene Kompetenzeinschätzungen (Marsh et al. 2017) ein bedeutsames theoretisches, als auch empirisches Korrelat sowohl für Implizite Theorien (Dweck und Leggett 1988; Dickhäuser et al. 2017) als auch für Wohlbefinden (Marsh et al. 2006) darstellen, wurde das Zusammenspiel aller drei Konstrukte bislang nicht betrachtet. Die bisher wenigen Studien die das Zusammenspiel von Impliziten Theorien und Wohlbefinden untersuchten konzentrierten sich (a) fast ausschließlich auf das Fehlen von negativen psychologischen Faktoren wie Stress und Depression (Burnette et al. 2020), (b) vernachlässigten die Multidimensionalität von Wohlbefinden (Kern et al. 2015; Seligman 2011), und (c) berücksichtigen nicht den schulischen Kontext sowie die Rolle von selbstbezogenen Kompetenzeinschätzungen. Die vorliegende Studie schließt diese Lücken, indem sie Zusammenhänge zwischen Impliziten Fähigkeitstheorien, akademischem Selbstkonzept und schulbezogenem Wohlbefinden von Schüler*innen untersucht.

\subsection{Implizite Fähigkeitstheorien und akademisches Selbstkonzept}

In ihrer ursprünglichen Bezeichnung werden Implizite Theorien als „core assumptions about the malleability of personal qualities" definiert (Yeager und Dweck 2012, S. 303). Je nach psychologischer Subdisziplin finden sich aber weitere Begriffe wie implizite Überzeugungen, world views, Mindsets, self-theories, Laientheorien oder naive Theorien, die aber alle grundsätzlich dasselbe Konstrukt beschreiben (Lüftenegger und Chen 2017a). Unabhängig von der Bezeichnung fokussieren Implizite Theorien oder ihre verwandten Begriffe immer auf zwei unterschiedliche Perspektiven: Menschen betrachten menschliche Eigenschaften wie Intelligenz, Fähigkeiten oder soziale Eigenschaften entweder als (1) unveränderliche Eigen- 
schaften (z.B. Menschen werden mit einer bestimmten Persönlichkeit geboren, die nicht verändert werden kann), oder (2) sie betrachten menschliche Eigenschaften als formbare Qualitäten (z. B. Menschen sind in der Lage, ihre grundlegende Fähigkeit, Mathematik zu betreiben, kontinuierlich zu verbessern). Dieser Glaube an die Formbarkeit (=Inkrementelle Theorie) oder Fixiertheit menschlicher Eigenschaften (=Entitätstheorie) ist nicht nur auf Selbstüberzeugungen beschränkt und kann auch Überzeugungen über andere Menschen, Kontexte oder Phänomene umfassen (z.B. Überzeugungen über Gruppen in Konflikten, Goldenberg et al. 2018). Empirische Studien im Schulkontext fokussierten bisher hauptsächlich auf Überzeugungen über Intelligenz (Blackwell et al. 2007; Dinger und Dickhäuser 2013; Yeager et al. 2019), Fähigkeiten (Chen und Tutwiler 2017; Lüftenegger et al. 2015) oder Persönlichkeitsaspekte (Yeager et al. 2013, 2014).

In sozial-kognitiven Ansätzen zu Motivation stellen Implizite Theorien in Leistungskontexten wichtige Antezedenzien von sogenannten Bedeutungssystemen (meaning systems; Dweck und Molden 2017) dar. Dabei handelt es sich um Kognitionen, die für Erleben und Verhalten in kompetenzrelevanten Situationen zentral sind. In diesen Bedeutungssystemen prägen Implizite Theorien Einstellungen und/ oder Ziele, die wiederum wichtige Outcomes wie Lernverhalten oder Leistung beeinflussen (Burnette et al. 2013). Im Schulkontext konnte mehrfach gezeigt werden, dass Entitätstheorien über Intelligenz mit Performanz(vermeidungs)zielen, Hilflosigkeit und negativen Einstellungen zu Anstrengung zusammenhängen, und dass diese Einstellungen und Ziele Leistung und Wahl von herausfordernden Kursen negativ prädizieren (Yeager und Dweck 2020). Eine zentrale Rolle in leistungsbezogenen Bedeutungssystemen spielen auch selbstbezogene Kompetenzeinschätzungen (Dweck und Leggett 1988). Während Implizite Fähigkeitstheorien die Überzeugungen über eigene Fähigkeiten als stabil oder veränderbar darstellen, geben erst die selbstbezogenen Kompetenzeinschätzungen, die u. a. in Form von Selbstkonzept (Shavelson et al. 1976) oder Selbstwirksamkeitsüberzeugungen (Bandura 1997) konzeptualisiert werden, Aufschluss darüber, ob Individuen glauben über die persönlichen Ressourcen zu verfügen um erfolgreich handeln zu können (Chen und Tutwiler 2017). Diesen theoretischen Annahmen entsprechend zeigten auch empirische Studien, dass Inkrementelle Fähigkeitstheorien positiv und Entitätstheorien negativ mit selbstbezogenen Kompetenzeinschätzungen zusammenhängen (Chen und Tutwiler 2017; Dickhäuser et al. 2017; Lüftenegger et al. 2015; Priess-Groben und Hyde 2017). Dementsprechend gehen wir auch für diese Studie davon aus, dass Inkrementelle Fähigkeitstheorien positiv mit akademischem Selbstkonzept zusammenhängen.

In Bedeutungssystemen nach Dweck und Molden (2017) sind Einschätzungen über eigene Kompetenzen als Verbindungsglied zwischen Impliziten Theorien und möglichen Outcomes wie Verhalten, Leistung oder Gesundheitsverhalten konzeptualisiert. Kaum empirisch untersucht wurde jedoch bisher, welche Rolle Inkrementelle Fähigkeitstheorien und die damit verbundenen Kompetenzeinschätzungen für (schulisches) Wohlbefinden haben. 


\subsection{Schulisches Wohlbefinden}

Wohlbefinden wird in der Literatur sehr unterschiedlich konzeptualisiert (Deci und Ryan 2008; Diener 1984). Dies trifft auch auf Operationalisierungen von schulischem Wohlbefinden zu, die von globaler Lebenszufriedenheit (OECD 2017), der Abwesenheit von Depression (Karvonen et al. 2018) bis hin zu Schul- und Lernklima (Hascher und Hagenauer 2010; Niclasen et al. 2018) reichen. Die fehlende Vergleichbarkeit dieser Befunde macht daher Forschungssynthesen als auch die Ableitung von spezifischen Maßnahmen schwierig. Diese Defizite sprechen für die Verwendung von ganzheitlicheren Rahmenmodellen, die die unterschiedlichen Aspekte von Wohlbefinden berücksichtigen.

Neuere Modelle aus der Positiven Psychologie (PERMA, Seligman 2011; EPOCH, Kern et al. 2015, 2016) verfolgen einen holistischeren Zugang und konzeptualisieren Wohlbefinden als ein multidimensionales Konstrukt, das sowohl hedonistische Komponenten (Fokus auf positiven Affekt) als auch Prozesse der Selbstverwirklichung und Potenzialentfaltung, d.h. eudaemonisches Wohlbefinden, beinhaltet (Ryan und Deci 2001; Deci und Ryan 2008). PERMA und EPOCH stellen dabei jeweils Akronyme der innerhalb der Modelle postulierten Komponenten von Wohlbefinden dar. PERMA fokussiert auf das Wohlbefinden von Erwachsenen entlang der Komponenten Positive Emotions, Engagement, Relationships, Meaning und Achievement (Seligman 2011). Das EPOCH-Modell (Kern et al. 2016) fokussiert auf das Wohlbefinden von Jugendlichen und besteht aus den fünf Komponenten Engagement, Perseverance, Optimism, Connectedness und Happiness. ${ }^{1}$ Da der jeweilige Kontext für das korrekte Verständnis von Wohlbefinden bedeutsam ist (Bradshaw et al. 2011), wurden die fünf Komponenten in aktuellen Publikationen für den Schulkontext spezifiziert (Buerger et al. 2021; Holzer et al. 2021). Engagement bezeichnet das völlige Aufgehen in und die Konzentration auf schulbezogene Aktivitäten und Aufgaben. Ein sehr hohes Maß an Engagement wird als „Flow“ (Csikszentmihalyi 1997) verstanden. Perseverance beschreibt das konsistente Anstreben von Zielen auch angesichts von Hindernissen. Hoffnung und Zuversicht für die schulbezogene Zukunft wird als Optimism bezeichnet. Connectedness umfasst befriedigende beidseitige Beziehungen und Freundschaften im Schulkontext. Happiness spiegelt stetige positive Stimmung und Zufriedenheit in der Schule wider. Engagement, Peserverance und Optimism sind dabei auf konkrete Aufgaben bezogen. Connectedness und Happiness fokussieren allgemein auf den schulischen Kontext.

Wohlbefinden und psychische Gesundheit wurden in den letzten Jahren auch mit Impliziten Theorien in Verbindung gebracht (Burnette et al. 2020; Yeager und Dweck 2020). Bezugnehmend auf Forschung zur kognitiven Verhaltenstherapie kann angenommen werden, dass inkrementelle Theorien die negativen Auswirkungen negativer Lebensereignisse abfedern. Dies geschieht unter anderem deshalb, weil maladaptive Kognitionen, wie der Glaube an die Unveränderlichkeit des eigenen Zustands psychologische Störungen begünstigen können (z.B. Beck 1987). Es ist

\footnotetext{
${ }^{1}$ Es werden die englischen Originalbegriffe verwendet da es für mehrere Dimensionen (wie z. B. Engagement, Happiness, Connectedness) keine eindeutigen bzw. passenden deutschen Übersetzungen gibt.
} 
daher naheliegend, dass eine inkrementelle Theorie mit einer geringeren psychischen Belastung verbunden sein sollte (Yeager 2017). Diese Befunde zeigten sich auch in einer aktuellen Meta-Analyse von Burnette et al. (2020), in der Inkrementelle Theorien von Jugendlichen negativ mit psychologischen Belastungen $(\mathrm{r}=-0,19$; 17 Studien) und positiv mit aktiven Bewältigungsstrategien ( $\mathrm{r}=0,19 ; 8$ Studien) zusammenhängen. Ähnliche Effekte zeigten sich auch für Entitätstheorien und psychologische Störungen bei Jugendlichen ( $r=0,25 ; 17$ Studien; Schleider et al. 2015). Der zugrunde liegende psychologische Mechanismus wurde in diesen Meta-Analysen allerdings nicht untersucht (Seo et al. 2021). Die direkten Effekte sind aber in etwa doppelt so groß wie die in aktuellen Meta-Analysen (Costa und Faria 2018; Sisk et al. 2018) gefundenen Zusammenhänge zwischen Inkrementellen Theorien und akademischer Leistung. Dies spricht dafür, den Forschungsfokus auch im akademischen Kontext stärker auf psychosoziale Outcomes zu richten. In empirischen Studien im Schulkontext zeigten sich kleine bis mittlere Zusammenhänge zwischen Impliziten Theorien über Intelligenz und unterschiedlichen Aspekten von Wohlbefinden. So waren beispielsweise bei chinesischen Schüler*innen Inkrementelle Intelligenztheorien positiv mit eudaemonischem Wohlbefinden und Lebenszufriedenheit verbunden (Chan 2012; Zeng et al. 2016). In einer weiteren Studie prädizierten Entitätstheorien negativen Affekt von philippinischen Sekundarschüler*innen in einer Längsschnittstudie (King 2017). In Stichproben mit australischen, US-amerikanischen, finnischen und chinesischen Jugendlichen fanden sich konsistent positive Zusammenhänge $(\mathrm{r}=0,1-0,3)$ zwischen Inkrementellen Theorien und den EPOCH Dimensionen (Kern et al. 2016; Park et al. 2020; Tang et al. 2019; Zeng et al. 2016; Zeng und Kern 2019). Diese Studien berücksichtigten allerdings den Schulkontext nicht in ihren Operationalisierungen und fokussierten fast ausschließlich auf Implizite Theorien über Intelligenz. Zusätzlich ist nicht klar, durch welchen psychologischen Mechanismus situationsübergreifende Überzeugungen auf hedonistische und eudaemonisches Aspekte des Wohlbefindens in Leistungskontexten, wirken. Folgt man theoretischen Überlegungen (Dweck und Leggett 1988; Chen und Tutwiler 2017) als auch empirischen Untersuchungen zu Bedeutungssystemen in Leistungskontexten (Burnette et al. 2013; Seo et al. 2021; Yeager und Dweck 2020) stellen selbstbezogene Kompetenzüberzeugen ein aussichtsreiches Konstrukt dar, welches den psychologischen Prozess vermitteln könnte.

Befunde über Zusammenhänge zwischen dem akademischen Selbstkonzept und Wohlbefinden im Schulkontext finden sich nur wenige. Eine erwähnenswerte Ausnahme bildet die Studie von Marsh und Kolleg*innen (2006), in der akademisches Selbstkonzept mit allgemeiner Lebenszufriedenheit $(r=0,27)$, positivem $(r=0,34)$ und negativem Affekt $(r=-0,32)$ korreliert war. Domänenspezifische Selbstkonzeptskalen für Mathematik und Deutsch zeigten ähnliche Muster bei etwas schwächeren Effekten. In den Validierungsstudien des domänenunspezifischen EPOCH Fragebogens zeigten sich positive Zusammenhänge von allen fünf EPOCH Komponenten mit selbsteingeschätzter Schulleistung $(r=0,29-0,53$; Kern et al. 2016) und Selbstwirksamkeit ( $r=0,51-0,59$; Zeng und Kern 2019). Auch bei diesen Studien fehlen jedoch schulbezogene Operationalisierungen von Wohlbefinden (für Ausnahmen für einzelne EPOCH Komponenten siehe z. B. Schnitzler et al. 2020 oder Usher et al. 2019). 
Obwohl selbstbezogene Kompetenzeinschätzungen ein bedeutsames theoretisches (Dweck und Leggett 1988) als auch empirisches Korrelat sowohl für Implizite Theorien (Chen und Tutwiler 2017; Dickhäuser et al. 2017; Diseth et al. 2014; Lüftenegger et al. 2015) als auch für Wohlbefinden (Marsh et al. 2006) darstellen, wurde ihre Rolle im Zusammenspiel aller drei Konstrukte bislang nicht untersucht.

\subsection{Forschungsanliegen und Hypothesen}

Die vorliegende Studie befasst sich mit dem Zusammenspiel von Impliziten schulischen Fähigkeitstheorien, akademischem Selbstkonzept und schulischem Wohlbefinden in der Sekundarstufe. Im postulierten konzeptuellen Bedeutungssystem gehen wir davon aus, dass die impliziten schulischen Fähigkeitstheorien von Schüler*innen direkte Effekte auf das akademische Selbstkonzept und die fünf EPOCH Komponenten des schulischen Wohlbefindens (Engagement, Peserverance, Optimism, Connectedness, Happiness) haben, sowie indirekte Effekte auf die Dimensionen schulischen Wohlbefindens, vermittelt über das akademische Selbstkonzept (siehe auch Abb. 1). Dementsprechend testen wir direkte Effekte der Impliziten Fähigkeitstheorien auf das akademische Selbstkonzept (Hypothese $(=\mathrm{H})$ 1), direkte Effekte des akademischen Selbstkonzepts auf die fünf Dimensionen des schulischen Wohlbefindens (H 2a-2e), direkte Effekte der Impliziten Fähigkeitstheorien auf schulbezogenes Wohlbefinden $(\mathrm{H} \mathrm{3a}-3 \mathrm{e})$ und indirekte Effekte von Impliziten Fähigkeitstheorien auf die Dimensionen des schulbezogenen Wohlbefindens, vermittelt über das akademische Selbstkonzept ( $\mathrm{H} 4 \mathrm{a}-4 \mathrm{e})$.

Basierend auf den zuvor dargelegten theoretischen Überlegungen und bisherigen empirischen Befunden stellen wir folgende Annahmen auf: Eine inkrementelle schulische Fähigkeitstheorie ist positiv mit dem akademischen Selbstkonzept verbunden (H 1). Zusätzlich nehmen wir positive Zusammenhänge zwischen akademischem Selbstkonzept und den drei aufgabenbezogenen Dimensionen des schulischen Wohlbefindens an (H 2a-2c). Zusätzlich untersuchen wir explorativ den Zusammenhang zwischen akademischem Selbstkonzept und Connectedness sowie Happiness (H 2d-2e). Inkrementelle schulische Fähigkeitstheorien sollten auch jeweils positiv

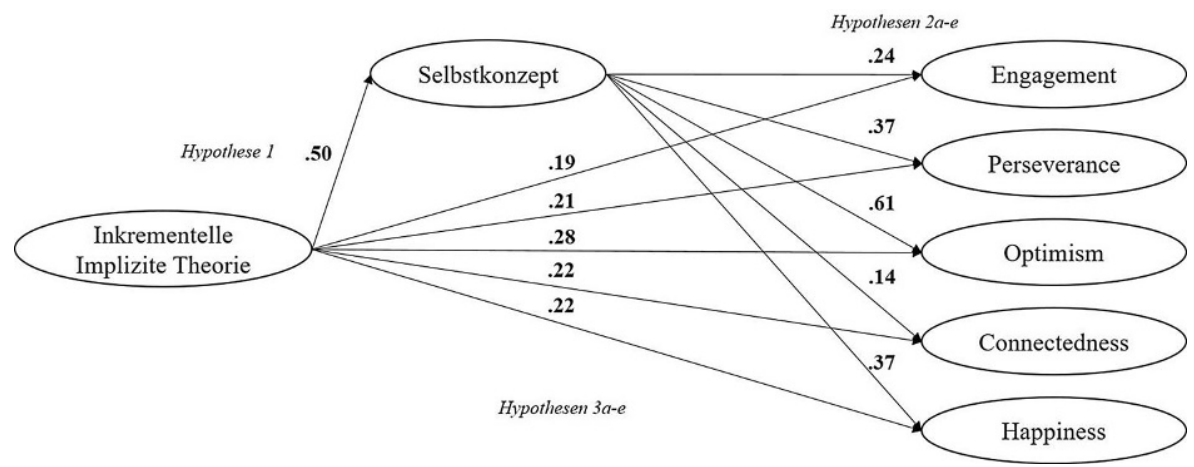

Abb. 1 Mediationsmodell mit direkten standardisierten Effekten für die untersuchten Hypothesen 1-3. Es zeigt ein Bedeutungssystem mit Impliziten Theorien, akademischem Selbstkonzept und den Zusammenhang mit 5 Dimensionen des schulischen Wohlbefindens 
(H 3a-3e) mit den fünf Dimensionen des schulischen Wohlbefindens zusammenhängen. Zuletzt untersuchen wir, ob indirekte Effekte von inkrementellen Fähigkeitstheorien auf die fünf Dimensionen des schulischen Wohlbefindens, mediiert durch akademisches Selbstkonzept, bestehen ( $\mathrm{H} 4 \mathrm{a}-4 \mathrm{e})$.

Frühere Befunde zum Wohlbefinden von Jugendlichen zeigen, dass ältere Jugendliche tendenziell ein geringeres Wohlbefinden erleben (Ronen et al. 2016). Daher haben wir in unserem Modell für Alter kontrolliert.

\section{Methoden}

\subsection{Stichprobe}

Die Stichprobe umfasste 1484 österreichische Schüler*innen (47,5\% männlich, $52,0 \%$ weiblich, 0,5\% divers) von 87 Klassen in 7 Wiener Schulen. Die Teilnehmer*innen waren im Durchschnitt 12,95 Jahre alt $(S D=2,10$; Median=13,00; Range $=10-19)$. Diese Studie ist Teil eines größeren Forschungsprojekts zu Wohlbefinden in der Schule (Holzer 2021). Die Teilnahme an der Studie war freiwillig und nur diejenigen, die aktiv ihre Zustimmung gaben, konnten auch mitmachen. Zusätzlich wurde das Einverständnis der Eltern zur Teilnahme an der Studie sowie zur Datennutzung eingeholt. Den Schüler*innen wurde die vollständige Anonymität ihrer Daten zugesichert. In Übereinstimmung mit den Vorgaben der Bildungsdirektion Wien wurden das Studiendesign und die Messinstrumente vor der Durchführung der Untersuchung von den jeweiligen Schulgemeinschaftsausschüssen genehmigt. Die teilnehmenden Schüler*innen füllten im Rahmen einer Unterrichtsstunde unter Supervision von geschulten Forschungsassistent*innen die Papier-Bleistift Fragebögen aus.

\subsection{Messinstrumente}

Schulisches Wohlbefinden wurde mit dem EPOCH-G-S gemessen (Buerger et al. 2021). Dieses Instrument wurde für Schüler*innen der Sekundarstufe entwickelt und erfasst mit 19 Items die fünf EPOCH Dimensionen im Schulkontext. Die fünf Subskalen sind Engagement (4 Items; Beispielitem: „Wenn ich in der Schule etwas Neues lerne, merke ich nicht, wie die Zeit vergeht."; Composite Reliability=0,72), Perseverance (4 Items; ,Wenn ich eine Schulaufgabe begonnen habe, bringe ich sie auch zu Ende.“; $C R=0,80$ ), Optimism (3 Items; ,Ich glaube, dass ich noch eine gute Schulzeit haben werde.“; $C R=0,69$ ), Connectedness (4 Items; ,Wenn mir etwas Gutes passiert, möchte ich in der Schule jemandem davon erzählen. “; $C R=0,72$ ) und Happiness (4 Items; ,Ich fühle mich wohl in der Schule.“; $C R=0,86$ ). Alle Fragen wurden auf einer fünfstufigen Ratingskala von ,stimmt gar nicht“ bis ,stimmt genau“ beantwortet.

Zur Messung des akademischen Selbstkonzepts verwendeten wir die Skala absolutes Selbstkonzept (SASK; Dickhäuser et al. 2002), welche insgesamt fünf Items umfasst. Die Fragen zur absoluten Repräsentation der eigenen Fähigkeiten werden 
dabei anhand von 5-stufigen semantischen Differentialen beantwortet (Beispiel: „Ich kann in der Schule ... wenig (1) ... viel (5)“; CR=0,82).

Zur Messung von inkrementellen Impliziten Fähigkeitstheorien im Schulkontext wurden vier Items der Skala von Lüftenegger und Kolleg*innen (2019) verwendet (für Diskussionen zur Dimensionalität Impliziter Theorien siehe z. B. Lüftenegger und Chen 2017a). Um dem Kontext unserer Forschungsfrage, d.h. dem allgemeinen Schulkontext, zu entsprechen, wurden die Items leicht angepasst und der Bezug zu einem konkreten Schulfach entfernt (Beispiel: „Wie viel ich in einem Schulfach kann, ist nicht festgelegt. Ich kann meine Fähigkeiten steigern."; $C R=0,75)$. Ein Item (,Ich kann in der Schule viel Neues dazulernen“) der ursprünglich aus 5 Fragen bestehenden Skala wurde nach der psychometrischen Analyse und aufgrund Bedenken bezüglich inhaltlicher Validität nach der Adaptierung ausgeschlossen.

\subsection{Datenanalyse}

Die Daten wurden mit SPSS 26 und Mplus 8.4 (Muthén und Muthén 2017) analysiert. Berechnet wurden kongenerische (=composite reliability; Raykov 2009) und tau-äquivalente Reliabilität (=Koeffizient Alpha; Cronbach 1951) sowie konfirmatorische Faktorenanalysen zur Testung der Faktorenstruktur der eingesetzten Skalen.

Um die Gefahr von „Commen Method Variance“ zu verringern, haben wir vorab versucht fähigkeits-, motivations- und aufgabenbezogene Faktoren zu minimieren (Podsakoff et al. 2003, 2012). Zusätzlich haben wir anhand des Harman-EinfaktorenTest (Podsakoff und Organ 1986) sowie eines gemeinsamen latenten Methodenfaktors (Liang et al. 2007) untersucht, ob eine Verzerrung durch common-method bias vorliegt. Beide Analysen sprechen dafür, dass ein common-method-bias kein großes Problem darzustellen scheint (für Details siehe Online Material).

Zur Prüfung der Forschungshypothesen wurden latente Mediationsanalysen durchgeführt um direkte Effekte von inkrementeller Fähigkeitstheorie (Prädiktor) auf das schulbezogene Wohlbefinden (Outcomes), direkte Effekte des akademischen Selbstkonzepts (Mediator) auf das schulbezogene Wohlbefinden, direkte Effekte der inkrementellen Fähigkeitstheorie auf das akademische Selbstkonzept sowie indirekte Effekte der inkrementellen Fähigkeitstheorie auf das schulische Wohlbefinden, vermittelt über das akademische Selbstkonzept, zu untersuchen. In Voranalysen zeigten sich kleine bis moderate statistisch signifikante Geschlechts- ( $r=0,05$ bis $0,10)$ und Alterseffekte $(r=-0,15$ bis $-0,29)$ im schulischen Wohlbefinden (Engagement, Perseverance, Optimism, Happiness) und im akademischen Selbstkonzept, die beide als Kovariaten ins Modell aufgenommen wurden. Zusätzlich wurde für Leistung (latenter Faktor aus den letzten Zeugnisnoten in den Hauptfächern Deutsch, Mathematik, Englisch) bei akademischem Selbstkonzept und schulischem Wohlbefinden kontrolliert. In allen Analysen wurde die hierarchische Datenstruktur (Schüler*innen genestet in Klassen) mit Hilfe von clusterrobusten Standardfehlern berücksichtigt (Bardach et al. 2020).

Bei allen Modellen wurde grundsätzlich ein robuster Maximum Likelihood Schätzer (MLR) verwendet. In einem Model mit ML Schätzung wurde zusätzlich die statistische Signifikanz der direkten und indirekten Effekte mit Hilfe von Bootstrapping-Konfidenzintervallen (10.000 Ziehungen) abgesichert. Es zeigten sich dabei 
keine Unterschiede in den Effekten im Vergleich zum Modell mit der MLR Schätzung. Diese $95 \%$ Konfidenzintervalle werden ebenfalls berichtet. Aufgrund der großen Stichprobe und der davon abhängigen Signifikanztests haben wir uns bei der Interpretation der Ergebnisse auch auf die Effektgrößen der Regressionsparameter fokussiert. Dabei folgten wir Cohen (1988), demzufolge standardisierte Werte um 0,10, 0,30 und 0,50 als kleine, moderate und große Effekte interpretiert werden.

Der Fit der untersuchten Modelle wurde mittels $\chi^{2}$-Test, CFI, TLI, RMSEA und SRMR bestimmt. Cutoff-Werte von CFI, TLI >0,95 und 0,90 und RMSEA, SRMR $<0,06$ und 0,08 sprechen für eine exzellente bzw. adäquate Modellanpassung (Hu und Bentler 1999). Der Anteil der fehlenden Werte war mit 0,1 bis $2 \%$ auf der ItemEbene sehr gering. Die fehlenden Werte wurde mit dem Full Information Maximum Likelihood-Ansatz in den jeweiligen Modellen geschätzt.

\section{Ergebnisse}

Die Ergebnisse der KFAs zeigte jeweils einen adäquaten Modell Fit für den EPOCHG-S $\left(\chi^{2}(142)=684,796 ; p<0,001 ; \mathrm{CFI}=0,934 ; \mathrm{TLI}=0,921 ; \mathrm{RMSEA}=0,051\right.$; SRMR $=0,045)$, für Inkrementelle Fähigkeitstheorien $\left(\chi^{2}(2)=6,839 ; p=0,003\right.$; $\mathrm{CFI}=0,994 ; \mathrm{TLI}=0,98 \mathrm{RMSEA}=0,040 ; \mathrm{SRMR}=0,012)$ und das akademische Selbstkonzept $\left(\chi^{2}(5)=67,520 ; p<0,001 ; \mathrm{CFI}=0,954\right.$; TLI =0,907; RMSEA = 0,092; SRMR =0,035). Bivariate latente Korrelationen, deskriptive Statistiken und Reliabilität für alle Variablen finden sich in Tab. 1. Das Mediationsmodell mit MLR Schätzung zeigte einen adäquaten Model Fit $\left(\chi^{2}(456)=1629,940 ; p<0,001 ; \mathrm{CFI}=0,916\right.$;

Tab. 1 Bivariate latente Korrelationen, Deskriptiv Statistiken und Reliabilität

\begin{tabular}{llllllll}
\hline & 1 & 2 & 3 & 4 & 5 & 6 & 7 \\
\hline 1. Engagement & - & & & & & & \\
2. Perseverance & $\mathbf{0 , 7 7}$ & - & & & & & \\
3. Optimism & $\mathbf{0 , 5 3}$ & $\mathbf{0 , 6 5}$ & - & & & & \\
4. Connectedness & $\mathbf{0 , 1 7}$ & $\mathbf{0 , 2 4}$ & $\mathbf{0 , 4 9}$ & - & & & \\
5. Happiness & $\mathbf{0 , 4 5}$ & $\mathbf{0 , 5 2}$ & $\mathbf{0 , 8 2}$ & $\mathbf{0 , 5 6}$ & - & & \\
6. Inkrementelle Theorie & $\mathbf{0 , 2 9}$ & $\mathbf{0 , 3 8}$ & $\mathbf{0 , 5 5}$ & $\mathbf{0 , 3 0}$ & $\mathbf{0 , 3 7}$ & - & $\mathbf{0 , 3 7}$ \\
7. Selbstkonzept & $\mathbf{0 , 3 5}$ & $\mathbf{0 , 5 3}$ & $\mathbf{0 , 7 3}$ & $\mathbf{0 , 2 8}$ & $\mathbf{0 , 4 6}$ & $\mathbf{0 , 4 7}$ & 5 \\
Anzahl Items & 4 & 4 & 3 & 4 & 4 & 4 & 3,56 \\
$M$ & 2,93 & 3,70 & 3,43 & 4,00 & 3,61 & 3,48 & 0,73 \\
SD & 0,86 & 0,82 & 0,87 & 0,80 & 0,93 & 0,49 & $-0,33$ \\
Skewness & 0,02 & $-0,41$ & $-0,35$ & $-0,95$ & $-0,50$ & $-1,05$ & 0,37 \\
Kurtosis & $-0,39$ & $-0,31$ & $-0,36$ & 0,42 & $-0,30$ & 1,29 & $1-5$ \\
Theoretischer Range & $1-5$ & $1-5$ & $1-5$ & $1-5$ & $1-5$ & $1-4$ & $1-5$ \\
Empirischer Range & $1-5$ & $1-5$ & $1-5$ & $1-5$ & $1-5$ & $1-4$ & 0,82 \\
CR & 0,71 & 0,80 & 0,65 & 0,72 & 0,86 & 0,75 & 0,82 \\
Koeffizient Alpha & 0,72 & 0,79 & 0,68 & 0,71 & 0,86 & 0,75 & 0,04 \\
ICC & 0,19 & 0,13 & 0,07 & 0,06 & 0,12 & 0,54 & \\
\hline Anmerkng & & & & & & \\
\end{tabular}

Anmerkung. $N=1484$. Statistisch signifikante Ergebnisse $(\alpha<0,05)$ sind fett markiert CR Composite Reliabilität 
Tab. 2 Ergebnisse der Mediationsanalyse

\begin{tabular}{|c|c|c|c|}
\hline & Est. (SE) & $95 \% \mathrm{CI}$ & Std. Est \\
\hline \multicolumn{4}{|l|}{ Direkte Effekte } \\
\hline Inkrementelle Theorie $\rightarrow$ Engagement & $0,28(0,07)$ & {$[0,15 ; 0,42]$} & $\mathbf{0 , 1 9}$ \\
\hline Inkrementelle Theorie $\rightarrow$ Perseverance & $\mathbf{0 , 3 0}(\mathbf{0 , 0 6})$ & {$[0,18 ; 0,43]$} & $\mathbf{0 , 2 1}$ \\
\hline Inkrementelle Theorie $\rightarrow$ Optimism & $0,44(0,07)$ & {$[0,31 ; 0,56]$} & $\mathbf{0 , 2 8}$ \\
\hline Inkrementelle Theorie $\rightarrow$ Connectedness & $0,23(0,05)$ & {$[0,13 ; 0,33]$} & $\mathbf{0 , 2 2}$ \\
\hline Inkrementelle Theorie $\rightarrow$ Happiness & $0,40(0,08)$ & {$[0,25 ; \mathbf{0 , 5 5}]$} & $\mathbf{0 , 2 2}$ \\
\hline Inkrementelle Theorie $\rightarrow$ Selbstkonzept & $0,56(0,06)$ & {$[0,45 ; 0,68]$} & $\mathbf{0 , 5 0}$ \\
\hline Selbstkonzept $\rightarrow$ Engagement & $0,24(0,06)$ & {$[0,13 ; 0,35]$} & 0,24 \\
\hline Selbstkonzept $\rightarrow$ Perseverance & $0,35(0,05)$ & {$[0,25 ; 0,45]$} & $\mathbf{0 , 3 7}$ \\
\hline Selbstkonzept $\rightarrow$ Optimism & $0,65(0,05)$ & {$[0,56 ; 0,75]$} & $\mathbf{0 , 6 1}$ \\
\hline Selbstkonzept $\rightarrow$ Connectedness & $0,10(0,04)$ & {$[0,02 ; 0,17]$} & $\mathbf{0 , 1 4}$ \\
\hline Selbstkonzept $\rightarrow$ Happiness & $0,44(0,06)$ & {$[0,34 ; \mathbf{0 , 5 5}]$} & $\mathbf{0 , 3 7}$ \\
\hline \multicolumn{4}{|l|}{ Indirekte Effekte } \\
\hline Inkrementelle Theorie $\rightarrow$ Selbstkonzept $\rightarrow$ Engagement & $0,13(0,04)$ & {$[0,07 ; 0,21]$} & $\mathbf{0 , 0 9}$ \\
\hline Inkrementelle Theorie $\rightarrow$ Selbstkonzept $\rightarrow$ Perseverance & $0,20(0,04)$ & {$[0,13 ; 0,28]$} & 0,14 \\
\hline Inkrementelle Theorie $\rightarrow$ Selbstkonzept $\rightarrow$ Optimism & $\mathbf{0 , 3 7}(\mathbf{0 , 0 4})$ & {$[0,29 ; 0,46]$} & $\mathbf{0 , 2 3}$ \\
\hline Inkrementelle Theorie $\rightarrow$ Selbstkonzept $\rightarrow$ Connectedness & $0,05(0,02)$ & {$[0,01 ; 0,10]$} & $\mathbf{0 , 0 5}$ \\
\hline Inkrementelle Theorie $\rightarrow$ Selbstkonzept $\rightarrow$ Happiness & $0,25(0,04)$ & {$[0,18 ; 0,33]$} & $\mathbf{0 , 1 4}$ \\
\hline \multicolumn{4}{|l|}{ Erklärte Varianz $\left(\mathbf{R}^{2}\right)$} \\
\hline Engagement & 0,18 & & \\
\hline Perseverance & 0,34 & & \\
\hline Optimism & 0,59 & & \\
\hline Connectedness & 0,12 & & \\
\hline Happiness & 0,30 & & \\
\hline Selbstkonzept & 0,43 & & \\
\hline
\end{tabular}

Anmerkung. Statistisch signifikante Ergebnisse $(\alpha<0,05)$ sind fett markiert Est. unstandardisierter Parameterschätzer, SE Standardfehler, Std. Est. Standardisierter Parameterschätzer, $95 \%$ CI $95 \%$ Bias-korrigierte Bootstrap Konfidenzintervalle

TLI=0,903; RMSEA=0,042; SRMR=0,048). Die Ergebnisse der Mediationsanalyse inklusive der Bootstrapping Intervalle für alle Effekte sind in Tab. 2 sowie in Abb. 1 dargestellt.

\subsection{Direkte Effekte zwischen Impliziter Theorie, akademischem Selbstkonzept und Wohlbefinden}

Es zeigte sich erwartungsgemäß ein starker positiver Zusammenhang zwischen Inkrementeller Fähigkeitstheorie und akademischem Selbstkonzept (H 1). Für akademisches Selbstkonzept zeigten sich wie erwartet direkte positive Zusammenhänge sowohl mit den drei aufgabenfokussierten Dimensionen Engagement, Perseverance und Optimism ( $\mathrm{H} \mathrm{2a-2c)} \mathrm{als} \mathrm{auch} \mathrm{mit} \mathrm{Connectedness} \mathrm{und} \mathrm{Happiness} \mathrm{(} \mathrm{H} \mathrm{2d-2e).} \mathrm{Im}$ Hinblick auf Effekte mit schulischem Wohlbefinden zeigten sich für Inkrementelle Fähigkeitstheorien die erwarteten positiven Zusammenhänge mit allen fünf Dimen- 
sionen (Engagement, Perseverance, Optimism, Connectedness, Happiness; H 3a-3e). Alle Ergebnisse der standardisierten direkten Effekte sind in Abb. 1 dargestellt.

\subsection{Indirekte Effekte von Impliziten Fähigkeitstheorien auf Wohlbefinden über akademisches Selbstkonzept}

Es zeigten sich die erwarteten indirekten positiven Effekte sowohl auf Engagement, Perseverance und Optimism $(\mathrm{H} 4 \mathrm{a}-4 \mathrm{c})$ als auch auf Connectedness und Happiness (H 2d-2e) vermittelt über Selbstkonzept. Die erklärte Varianz im Mediationsmodell lag für Optimism bei 59\%, für akademisches Selbstkonzept bei 43\%, für Perseverance bei $34 \%$, für Happiness bei $30 \%$, für Engagement bei $18 \%$ und für Connectedness bei $12 \%$.

\section{Diskussion}

Die vorliegende Studie untersuchte den Zusammenhang von Impliziten schulischen Fähigkeitstheorien mit akademischem Selbstkonzept und den fünf EPOCH Komponenten des schulischen Wohlbefindens, sowie indirekte Effekte von Impliziten Theorien auf das aufgabenbezogene schulische Wohlbefinden, vermittelt über das akademische Selbstkonzept. Durch den Einsatz eines multidimensionalen Messinstruments war es möglich, differenzierte Aussagen zu Teilaspekten des schulbezogenen Wohlbefindens von Schüler*innen im Sekundarbereich zu untersuchen. Dabei zeigte sich hypothesenkonform, dass eine inkrementelle schulische Fähigkeitstheorie positiv mit akademischem Selbstkonzept (H 1) verbunden ist. Dies bestätigt grundlegende theoretische Annahmen (Dweck und Leggett 1988) und empirische Befunde, die Implizite Fähigkeitstheorien mit selbstbezogenen Kompetenzeinschätzungen verbunden sehen (Burnette et al. 2013; Chen und Tutwiler 2017).

Für das akademische Selbstkonzept wiederum zeigten sich erwartungskonforme positive Zusammenhänge mit Engagement, Perseverance und Optimism ( $\mathrm{H} \mathrm{2a-2c).}$ Hier zeigen sich jeweils große Effekte mit allen drei aufgabenbezogenen Komponenten schulischen Wohlbefindens. Diese domänenspezifischen Effekte sind durchwegs größer als in vorangegangen Studien, in denen allgemeine Lebenszufriedenheit, Affekt oder die allgemeinen EPOCH Skalen als Indikatoren für Wohlbefinden berücksichtigt wurden (Marsh et al. 2006; Kern et al. 2016).

Wie erwartet hängen inkrementelle schulische Fähigkeitstheorien jeweils positiv mit den fünf Komponenten des schulischen Wohlbefindens zusammen ( $\mathrm{H} \mathrm{3a-3e)}$. Es zeigt sich damit ein konsistentes Muster aus mittleren Effekten von Inkrementellen Fähigkeitstheorien auf aufgabenbezogene, zukunftsorientierte, affektive und soziale Aspekte von schulischem Wohlbefinden. Dies erweitert bisherige Befunde, die auch positive, jedoch kleinere Effekte bei globalen und domänenunspezifischen Operationalisierungen wie Lebenszufriedenheit und Affekt fanden (Chan 2012; Park et al. 2020; Tang et al. 2019; Zeng et al. 2016).

Erwartungsgemäß zeigten sich zudem indirekte positive Effekte von Inkrementellen Fähigkeitstheorien auf Engagement, Perseverance und Optimism, vermittelt über das akademische Selbstkonzept $(\mathrm{H} 4 \mathrm{a}-4 \mathrm{c})$. Dieser Befund zeigt auch die wichtige 
Rolle des Selbstkonzepts im hier untersuchten Bedeutungssystem. Die erklärte Varianz der fünf Outcomes belegt, dass das untersuchte Bedeutungssystem mit $59 \%$ bzw. $34 \%$ eine große Erklärungsmacht für Optimism bzw. Peserverance hat. Dies spricht dafür, dass inkrementelle Fähigkeitstheorien und Kompetenzeinschätzungen, sowie ihr Zusammenspiel, insgesamt bedeutsam für das konsistente Anstreben von Zielen bei gleichzeitiger Zuversicht für die schulbezogene Zukunft sind. Im Vergleich dazu zeigt sich für Connectedness, und damit den sozialen Aspekt schulischen Wohlbefindens, nur eine geringe Erklärungsmacht (10\% erklärte Varianz). Dies erscheint auch durchaus plausibel, da soziale Aspekte im Schulkontext keine nahen primären Konsequenzen von selbstbezogenen Kompetenzeinschätzungen darstellen (Bardach et al. 2021).

Das angenommene Bedeutungssystem und die Auswirkungen auf das schulische Wohlbefinden konnte in der untersuchten österreichischen Sekundarstufenstichprobe bestätigt werden. Die Ergebnisse der statistischen Analysen legen nahe, dass alle postulierten Hypothesen angenommen werden können. Das spricht für eine gute Anbindung der untersuchten Zusammenhänge an bisherige Forschungsbefunde und damit auch für die Robustheit der Ergebnisse. Zukünftige Studien könnten das untersuchte Bedeutungssystem noch um weitere Mediatoren wie selbstbezogene Ziele (Mastery, Performanz), Attributionen (Hilflosigkeit) oder negative Emotionen erweitern (Burnette et al. 2013; Yeager und Dweck 2020).

Trotz der Stärken der vorliegenden Studie, einschließlich einer großen Stichprobe, der Verwendung von differenzierten Messinstrumenten und der Prüfung eines neuen Bedeutungssystems für schulisches Wohlbefinden, gibt es auch Limitationen. Das nicht-experimentelle querschnittliche Design der Studie schränkt kausale Interpretationen der Ergebnisse ein. In der vorliegenden Studie wurden, basierend auf theoretischen Annahmen und empirischen Befunden (Dweck und Molden 2017; Yeager und Dweck 2020), Implizite Theorien als Ausgangspunkt des Bedeutungssystems mit Auswirkungen auf das schulische Wohlbefinden konzeptualisiert. Das spezifizierte Mediationsmodell bildet diese Zusammenhänge lediglich durch Querschnittsdaten ab. Die Annahme eines kausalen Wirkmechanismus ist rein auf theoretischer Ebene begründet. Aus Sicht der positiven Psychologie (Seligman 2011) und der Selbstbestimmungstheorie (Deci und Ryan 2008), die Wohlbefinden als einen Prozess konzeptualisieren, der Entwicklung fördert, wären allerdings auch Effekte von einzelnen Aspekten des Wohlbefindens auf Implizite Theorien denkbar (Park et al. 2020). Die Entwicklung und Stabilität des untersuchten Bedeutungssystems sowie bidirektionale Effekte und Interaktionen innerhalb dieses Bedeutungssystems sollten in längsschnittlichen Designs genauer untersucht werden. Eine weitere Limitation betrifft den Umstand, dass die Studie an einer Ad-hoc-Stichprobe von Sekundarschüler*innen in Wien durchgeführt wurde, die trotz ihrer Größe aufgrund des rein urbanen Kontexts nicht repräsentativ für den deutschsprachigen Raum ist. Schlussendlich sei angemerkt, dass alle erhobenen Daten aus der gleichen Quelle, einem standardisierten Selbstberichtfragebogen, stammen. Obwohl wir versucht haben, systematische Effekte beim Ausfüllen zu minimieren (Podsakoff et al. 2003, 2012), zeigten sich für die Skalen Optimism und Happiness Anzeichen eines common method bias (Podsakoff und Organ 1986). 
Für die Praxis lässt sich aus unseren Ergebnissen vorsichtig ableiten, dass Interventionen zur Förderung von inkrementellen Impliziten Theorien (Yeager et al. 2019) neben Leistung auch auf verschiedene Aspekte des schulbezogenen Wohlbefindens wirken könnten. Diese Mindset Interventionen erwiesen sich bisher aber hauptsächlich in Stichproben mit US-amerikanischen Schüler*innen als wirksam (Yeager et al. 2016). Möglichkeiten für solche Interventionen umfassen aber auch Reattributionstrainings (siehe z. B. Ziegler und Schober 2001). Diese streben realistische Einschätzungen der Ursachen von Leistungshandlungen an. In pädagogischen Kontexten hat sich hierfür insbesondere attributionales Feedback als praktikabel zur Förderung von inkrementellen Impliziten Theorien bei Schüler*innen erwiesen (z. B. Dresel und Ziegler 2006). Die Durchführung von Interventionsstudien und die damit einhergehende Messung von Veränderungen im schulischen Wohlbefinden, einhergehend mit Veränderungen in Impliziten Theorien sowie dem akademischen Selbstkonzept, würde die in dieser Studie angenommenen Mechanismen weiter empirisch untermauern.

Zusatzmaterial online Zusätzliche Informationen sind in der Online-Version dieses Artikels (https://doi. org/10.1007/s42010-021-00130-x) enthalten.

Funding Open access funding provided by University of Vienna.

Open Access Dieser Artikel wird unter der Creative Commons Namensnennung 4.0 International Lizenz veröffentlicht, welche die Nutzung, Vervielfältigung, Bearbeitung, Verbreitung und Wiedergabe in jeglichem Medium und Format erlaubt, sofern Sie den/die ursprünglichen Autor(en) und die Quelle ordnungsgemäß nennen, einen Link zur Creative Commons Lizenz beifügen und angeben, ob Änderungen vorgenommen wurden.

Die in diesem Artikel enthaltenen Bilder und sonstiges Drittmaterial unterliegen ebenfalls der genannten Creative Commons Lizenz, sofern sich aus der Abbildungslegende nichts anderes ergibt. Sofern das betreffende Material nicht unter der genannten Creative Commons Lizenz steht und die betreffende Handlung nicht nach gesetzlichen Vorschriften erlaubt ist, ist für die oben aufgeführten Weiterverwendungen des Materials die Einwilligung des jeweiligen Rechteinhabers einzuholen.

Weitere Details zur Lizenz entnehmen Sie bitte der Lizenzinformation auf http://creativecommons.org/ licenses/by/4.0/deed.de.

\section{Literatur}

Bandura, A. (1997). Self-efficacy: the exercise of control. New York: Freeman.

Bardach, L., Yanagida, T., \& Lüftenegger, M. (2020). Studying classroom climate effects in the context of multi-level structural equation modelling: An application-focused theoretical discussion and empirical demonstration. International Journal of Research \& Method in Education, 43(4), 348-363. https://doi.org/10.1080/1743727X.2020.1791071.

Bardach, L., Daumiller, M., \& Lüftenegger, M. (2021). Multiple social and academic achievement goals: Students' goal profiles and their linkages. The Journal of Experimental Education, angenommen.

Beck, A. T. (1987). Cognitive models of depression. Journal of Cognitive Psychotherapy, 1, 5-37.

Blackwell, L.S., Trzesniewski, K.H., \& Dweck, C.S. (2007). Implicit theories of intelligence predict achievement across an adolescent transition: A longitudinal study and an Intervention. Child Development, 78(1), 246-263. https://doi.org/10.1111/j.1467-8624.2007.00995.x.

Bradshaw, J., Keung, A., Rees, G., \& Goswami, H. (2011). Children's subjective well-being: International comparative perspectives. Children and Youth Services Review, 33(4), 548-556. https://doi.org/10. 1016/j.childyouth.2010.05.010. 
Buerger, S., Holzer, J., Yanagida, T., Schober, B., \& Spiel, C. (2021). The German version of the EPOCH measure of adolescent well-being adapted for the school context (EPOCH-G-S) - A Validation Study. Educational Assessment, Evaluation and Accountability, ,angenommen. Manuskript in Überarbeitung.

Burnette, J.L., Knouse, L.E., Vavra, D. T., O’Boyle, E., \& Brooks, M. A. (2020). Growth mindsets and psychological distress: a meta-analysis. Clinical Psychology Review, 77, 101816. https://doi.org/10. 1016/j.cpr.2020.101816.

Burnette, J.L., O'Boyle, E.H., VanEpps, E.M., Pollack, J.M., \& Finkel, E. J. (2013). Mind-sets matter: A meta-analytic review of implicit theories and self-regulation. Psychological Bulletin, 139(3), 655-701. https://doi.org/10.1037/a0029531.

Chan, D. W. (2012). Life satisfaction, happiness, and the growth mindset of healthy and unhealthy perfectionists among Hong Kong Chinese gifted students. Roeper Review, 34(4), 224-233. https://doi.org/ 10.1080/02783193.2012.715333.

Chen, J.A., \& Tutwiler, S. M. (2017). Implicit theories of ability and self-efficacy. Zeitschrift für Psychologie, 225(2), 127-136. https://doi.org/10.1027/2151-2604/a000289.

Cohen, J. (1988). Statistical power analysis for the behavioral sciences. Hillsdale, NJ: Erlbaum.

Costa, A., \& Faria, L. (2018). Implicit theories of intelligence and academic achievement: a meta-analytic review. Frontiers in Psychology, 9, 829. https://doi.org/10.3389/fpsyg.2018.00829.

Cronbach, L. (1951). Coefficient alpha and the internal structure of tests. Psychometrika, 16, 297-334. https://doi.org/10.1007/BF02310555.

Csikszentmihalyi, M. (1997). Finding flow: the psychology of engagement with everyday life. New York: Basic Books.

Dahl, R.E., Allen, N.B., Wilbrecht, L., \& Suleiman, A.B. (2018). Importance of investing in adolescence from a developmental science perspective. Nature, 554(7693), 441-450. https://doi.org/10. 1038/nature25770.

Deci, E.L., \& Ryan, R.M. (2008). Hedonia, eudaimonia, and well-being: An introduction. Journal of Happiness Studies, 9(1), 1-11. https://doi.org/10.1007/s10902-006-9018-1.

Dickhäuser, O., Janke, S., Praetorius, A.-K., \& Dresel, M. (2017). The effects of teachers' reference norm orientations on students' implicit theories and academic self-concepts. Zeitschrift für Pädagogische Psychologie, 31(3-4), 205-219. https://doi.org/10.1024/1010-0652/a000208.

Dickhäuser, O., Schöne, C., Spinath, B., \& Stiensmeier-Pelster, J. (2002). Die Skalen zum akademischen Selbstkonzept. Zeitschrift für Differentielle und Diagnostische Psychologie, 23, 393-405. https://doi. org/10.1024//0170-1789.23.4.393.

Diener, E. (1984). Subjective well-being. Psychological Bulletin, 95, 542-575. https://doi.org/10.1037/ 0033-2909.95.3.542.

Dinger, F.C., \& Dickhäuser, O. (2013). Does implicit theory of intelligence cause achievement goals? Evidence from an experimental study. International Journal of Educational Research, 61, 38-47. https://doi.org/10.1016/j.ijer.2013.03.008.

Diseth, A., Meland, E., \& Breidablik, H.J. (2014). Self-beliefs among students: Grade level and gender differences in self-esteem, self-efficacy and implicit theories of intelligence. Learning and Individual Differences, 35, 1-8. https://doi.org/10.1016/j.lindif.2014.06.003.

Dresel, M., \& Ziegler, A. (2006). Langfristige Förderung von Fähigkeitsselbstkonzept und impliziter Fähigkeitstheorie durch computerbasiertes attributionales Feedback. Zeitschrift Für Pädagogische Psychologie, 20(1/2), 49-63. https://doi.org/10.1024/1010-0652.20.12.49.

Dweck, C. S. (2006). Mindset: the new psychology of success. New York: Random House.

Dweck, C. S. (2007). The perils and promises of praise. Educational Leadership, 65(2), 34-39.

Dweck, C.S., \& Leggett, E. L. (1988). A social-cognitive approach to motivation and personality. Psychological Review, 95(2), 256. https://doi.org/10.1037/0033-295X.95.2.256.

Dweck, C. S., \& Molden, D.C. (2017). Mindsets: Their impact on competence motivation and acquisition. In A. J. Elliot, C. S. Dweck \& D. S. Yeager (Hrsg.), Handbook of competence and motivation: theory and application (2. Aufl. S. 135-154). New York: Guilford.

Dweck, C. S., \& Yeager, D.S. (2019). Mindsets: A view from two eras. Perspectives on Psychological Science, 14(3), 481-496. https://doi.org/10.1177/1745691618804166.

Goldenberg, A., Cohen-Chen, S., Goyer, J.P., Dweck, C. S., Gross, J. J., \& Halperin, E. (2018). Testing the impact and durability of a group malleability intervention in the context of the Israeli-Palestinian conflict. Proceedings of the National Academy of Sciences, 115(4), 696-701. https://doi.org/10.1073/ pnas. 1706800115 .

Hascher, T. (2010). Learning and emotion: Perspectives for theory and research. European Educational Research Journal, 9(1), 13-28. https://doi.org/10.2304/eerj.2010.9.1.13. 
Hascher, T., \& Hagenauer, G. (2010). Schulisches Wohlbefinden im Jugendalter - Verläufe und Einflussfaktoren. In A. Ittel, H. Merkens \& L. Stecher (Hrsg.), Jahrbuch Jugendforschung (S. 15-45). Wiesbaden: VS.

Holzer, J. (2021). Well-being and Motivation in the Classroom. https://doi.org/10.17605/OSF.IO/5UMNP.

Holzer, J., Bürger, S., Lüftenegger, M., \& Schober, B. (2021). Revealing associations between students' school-related well-being, achievement goals, and academic achievement. Manuscript under review.

Howell, A. J. (2009). Flourishing: Achievement-related correlates of students' well-being. The Journal of Positive Psychology, 4(1), 1-13. https://doi.org/10.1080/17439760802043459.

Hu, L., \& Bentler, P. M. (1999). Cutoff criteria for fit indexes in covariance structure analysis: conventional criteria versus new alternatives. Structural Equation Modeling: A Multidisciplinary Journal, 6(1), 1-55. https://doi.org/10.1080/10705519909540118.

Karvonen, S., Tokola, K., \& Rimpelä, A. (2018). Well-being and academic achievement: differences between schools from 2002 to 2010 in the Helsinki metropolitan area. Journal of School Health, 88(11), 821-829. https://doi.org/10.1111/josh.12691.

Kern, M. L., Benson, L., Steinberg, E. A., \& Steinberg, L. (2016). The EPOCH measure of adolescent wellbeing. Psychological Assessment, 28(5), 586-597. https://doi.org/10.1037/pas0000201.

Kern, M. L., Waters, L.E., Adler, A., \& White, M. A. (2015). A multidimensional approach to measuring well-being in students: Application of the PERMA framework. The Journal of Positive Psychology, 10(3), 262-271. https://doi.org/10.1080/17439760.2014.936962.

King, R. B. (2017). A fixed mindset leads to negative affect: The relations between implicit theories of intelligence and subjective well-being. Zeitschrift für Psychologie, 225(2), 137-145. https://doi.org/ 10.1027/2151-2604/a000290.

Liang, H., Saraf, N., Hu, Q., Xue, Y. (2007). Assimilation of Enterprise Systems: The Effect of Institutional Pressures and the Mediating Role of Top Management. MIS Quarterly, 31(1), 59-87. https://doi.org/ $10.2307 / 25148781$.

Lüftenegger, M., \& Chen, J. A. (2017a). Conceptual Issues and Assessment of Implicit Theories. Zeitschrift Für Psychologie, 225(2), 99-106. https://doi.org/10.1027/2151-2604/a000286.

Lüftenegger, M., \& Chen, J.A. (2017b). Implicit theories: International perspectives from multiple domains. Zeitschrift Für Psychologie, 225(2), 97-98. https://doi.org/10.1027/2151-2604/a000285.

Lüftenegger, M., Bardach, L., Bergsmann, E., Schober, B., \& Spiel, C. (2019). A citizen science approach to measuring students' achievement goals. International Journal of Educational Research, 95, 36-51. https://doi.org/10.1016/j.ijer.2019.03.003.

Lüftenegger, M., Kollmayer, M., Bergsmann, E., Jöstl, G., Spiel, C., \& Schober, B. (2015). Mathematically gifted students and high achievement: The role of motivation and classroom structure. High Ability Studies, 26(2), 227-243. https://doi.org/10.1080/13598139.2015.1095075.

Marsh, H. W., Martin, A. J., Yeung, A. S., \& Craven, R. G. (2017). Competence self-perceptions. In A. J. Elliot, C.S. Dweck \& D.S. Yeager (Hrsg.), Handbook of competence and motivation (2. Aufl. S. 85-134). New York: Guilford.

Marsh, H.W., Trautwein, U., Lüdtke, O., Koller, O., \& Baumert, J. (2006). Integration of multidimensional self-concept and core personality constructs: Construct validation and relations to well-being and achievement. Journal of Personality, 74(2), 403-456. https://doi.org/10.1111/j.1467-6494.2005. 00380.x.

Muthén, L.K., \& Muthén, B.O. (2017). Mplus user's guide (8. Aufl.). Los Angeles, CA: Muthén \& Muthén.

Niclasen, J., Keilow, M., \& Obel, C. (2018). Psychometric properties of the Danish student well-being questionnaire assessed in $>250,000$ student responders. Scandinavian Journal of Public Health, 46(8), 877-885. https://doi.org/10.1177/1403494818772645.

OECD (2017). Bildung auf einen Blick 2017: OECD-Indikatoren. Bielefeld: Bertelsmann. https://doi.org/ 10.1787/eag-2017-de.

OECD (2019). PISA 2018 results (Volume III): What school life means for students' lives. OECD Publishing. https://doi.org/10.1787/acd78851-en.

Park, D., Tsukayama, E., Yu, A., \& Duckworth, A. L. (2020). The development of grit and growth mindset during adolescence. Journal of Experimental Child Psychology, 198, 104889. https://doi.org/10.1016/ j.jecp.2020.104889.

Podsakoff, P. M., \& Organ, D. W. (1986). Self-reports in organizational research: Problems and prospects. Journal of Management, 12(4), 531-544.

Podsakoff, P. M., MacKenzie, S. B., Lee, J.-Y., \& Podsakoff, N. P. (2003). Common method biases in behavioral research: A critical review of the literature and recommended remedies. Journal of Applied Psychology, 88(5), 879-903. https://doi.org/10.1037/0021-9010.88.5.879. 
Podsakoff, P. M., MacKenzie, S. B., \& Podsakoff, N.P. (2012). Sources of method bias in social science research and recommendations on how to control it. Annual Review of Psychology, 63, 539-569. https://doi.org/10.1146/annurev-psych-120710-100452.

Priess-Groben, H. A., \& Hyde, J.S. (2017). Implicit theories, expectancies, and values predict mathematics motivation and behavior across high school and college. Journal of Youth and Adolescence, 46(6), 1318-1332. https://doi.org/10.1007/s10964-016-0579-y.

Raykov, T. (2009). Evaluation of scale reliability for unidimensional measures using latent variable modeling. Measurement and Evaluation in Counseling and Development, 42(3), 223-232. https://doi.org/ 10.1177/0748175609344096.

Ronen, T., Hamama, L., Rosenbaum, M., \& Mishely-Yarlap, A. (2016). Subjective well-being in adolescence: The role of self-control, social support, age, gender, and familial crisis. Journal of Happiness Studies, 17(1), 81-104. https://doi.org/10.1007/s10902-014-9585-5.

Ryan, R. M., \& Deci, E.L. (2001). On happiness and human potentials: A review of research on hedonic and eudaimonic well-being. Annual Review of Psychology, 52(1), 141-166. https://doi.org/10.1146/ annurev.psych.52.1.141.

Schleider, J.L., Abel, M.R., \& Weisz, J.R. (2015). Implicit theories and youth mental health problems: A random-effects meta-analysis. Clinical Psychology Review, 35, 1-9. https://doi.org/10.1016/j.cpr. 2014.11.001.

Schnitzler, K., Holzberger, D., \& Seidel, T. (2020). All better than being disengaged: Student engagement patterns and their relations to academic self-concept and achievement. European Journal of Psychology of Education. https://doi.org/10.1007/s10212-020-00500-6.

Seligman, M.E.P. (2011). Flourish: A visionary new understanding of happiness and well-being. New York: Free Press.

Seo, E., Lee, H. Y., Jamieson, J.P., Reis, H., Josephs, R. A., Beevers, C. G., \& Yeager, D. S. (2021). Trait attributions and threat appraisals explain why an entity theory of personality predicts greater internalizing symptoms during adolescence. Development and Psychopathology. https://doi.org/10.1017/ S0954579420001832.

Shavelson, R. J., Hubner, J. J., \& Stanton, G. C. (1976). Self-concept: Validation of construct interpretations. Review of Educational Research, 46(3), 407-441. https://doi.org/10.3102/00346543046003407.

Sisk, V.F., Burgoyne, A.P., Sun, J., Butler, J.L., \& Macnamara, B. N. (2018). To what extent and under which circumstances are growth mind-sets important to academic achievement? Two meta-analyses. Psychological Science, 29(4), 549-571. https://doi.org/10.1177/0956797617739704.

Tang, X., Wang, M.-T., Guo, J., \& Salmela-Aro, K. (2019). Building grit: The longitudinal pathways between mindset, commitment, grit, and academic outcomes. Journal of Youth and Adolescence, 48(5), 850-863. https://doi.org/10.1007/s10964-019-00998-0.

Usher, E. L., Li, C. R., Butz, A. R., \& Rojas, J.P. (2019). Perseverant grit and self-efficacy: Are both essential for children's academic success? Journal of Educational Psychology, 111(5), 877-902. https:// doi.org/10.1037/edu0000324.

West, M. R., Buckley, K., Krachman, S. B., \& Bookman, N. (2018). Development and implementation of student social-emotional surveys in the CORE Districts. Journal of Applied Developmental Psychology, 55, 119-129. https://doi.org/10.1016/j.appdev.2017.06.001.

Yeager, D.S. (2017). Dealing with social difficulty during adolescence: the role of implicit theories of personality. Child Development Perspectives, 11(3), 196-201. https://doi.org/10.1111/cdep.12234.

Yeager, D.S., \& Dweck, C.S. (2012). Mindsets that promote resilience: When students believe that personal characteristics can be developed. Educational Psychologist, 47(4), 302-314. https://doi.org/10. $1080 / 00461520.2012 .722805$.

Yeager, D. S., \& Dweck, C. S. (2020). What can be learned from growth mindset controversies? American Psychologist, 75(9), 1269-1284. https://doi.org/10.1037/amp0000794.

Yeager, D.S., Dahl, R.E., \& Dweck, C.S. (2018). Why interventions to influence adolescent behavior often fail but could succeed. Perspectives on Psychological Science, 13(1), 101-122. https://doi.org/ $10.1177 / 1745691617722620$.

Yeager, D. S., Hanselman, P., Walton, G. M., Murray, J.S., Crosnoe, R., Muller, C., Tipton, E., Schneider, B., Hulleman, C. S., Hinojosa, C. P., Paunesku, D., Romero, C., Flint, K., Roberts, A., Trott, J., Iachan, R., Buontempo, J., Yang, S. M., Carvalho, C. M., Hahn, P. R., Gopalan, M., Mhatre, P., Ferguson, R., Duckworth, A. L., \& Dweck, C.S. (2019). A national experiment reveals where a growth mindset improves achievement. Nature, 573(7774), 364-369. https://doi.org/10.1038/s41586-019-1466-y.

Yeager, D. S., Johnson, R., Spitzer, B. J., Trzesniewski, K. H., Powers, J., \& Dweck, C. S. (2014). The farreaching effects of believing people can change: Implicit theories of personality shape stress, health, 
and achievement during adolescence. Journal of Personality and Social Psychology, 106(6), 867-884. https://doi.org/10.1037/a0036335.

Yeager, D.S., Miu, A.S., Powers, J., \& Dweck, C. S. (2013). Implicit theories of personality and attributions of hostile intent: A meta-analysis, an experiment, and a longitudinal intervention. Child Development, 84(5), 1651-1667. https://doi.org/10.1111/cdev.12062.

Yeager, D. S., Romero, C., Paunesku, D., Hulleman, C. S., Schneider, B., Hinojosa, C., Lee, H. Y., O’Brien, J., Flint, K., Roberts, A., Trott, J., Greene, D., Walton, G. M., \& Dweck, C.S. (2016). Using design thinking to improve psychological interventions: The case of the growth mindset during the transition to high school. Journal of Educational Psychology, 108(3), 374-391. https://doi.org/10.1037/ edu0000098.

Zeng, G., \& Kern, M.L. (2019). The Chinese EPOCH measure of adolescent wellbeing: Further testing of the psychometrics of the measure. Frontiers in Psychology, 10, 1457. https://doi.org/10.3389/fpsyg. 2019.01457.

Zeng, G., Hou, H., \& Peng, K. (2016). Effect of growth mindset on school engagement and psychological well-being of Chinese primary and middle school students: The mediating role of resilience. Frontiers in Psychology, 7, 1873. https://doi.org/10.3389/fpsyg.2016.01873.

Ziegler, A., \& Schober, B. (2001). Theoretische Grundlagen und praktische Anwendungen von Reattributionstrainings. Regensburg: Roderer. 